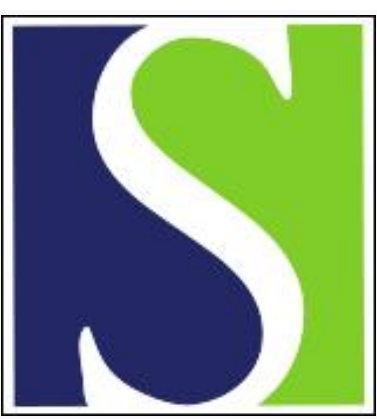

Scand J Work Environ Health 2011;37(1):62-69

https://doi.org/10.5271/sjweh.3122

Published online: 30 Sep 2010, Issue date: Jan 2011

A phase-advanced, extended sleep paradigm to increase sleep duration among early-morning shift workers: a preliminary investigation

by Yeung J, Sletten TL, Rajaratnam SM

Affiliation: School of Psychology and Psychiatry, Monash University, Building 17, Clayton Campus, Victoria 3800, Australia. shantha.rajaratnam@med.monash.edu.au

Refers to the following texts of the Journal: 1998;24 suppl 3:49-54 2010;36(2):96-108 1991;17(5):330-336

Key terms: actigraphy; circadian; extended sleep; phase advance; shift work; shift worker; sleep; wake maintenance zone

This article in PubMed: www.ncbi.nlm.nih.gov/pubmed/20882266 


\title{
A phase-advanced, extended sleep paradigm to increase sleep duration among early-morning shift workers: a preliminary investigation
}

\author{
by Jenny Yeung BA(Hons), ${ }^{1}$ Tracey L Sletten PhD, ${ }^{1}$ Shantha M Rajaratnam PhD ${ }^{1}$
}

Yeung J, Sletten TL, Rajaratnam SM. A phase-advanced, extended sleep paradigm to increase sleep duration
among early-morning shift workers: a preliminary investigation. Scand J Work Environ Health. 2011;37(1):62-69

Objective In this preliminary study, using a within-subjects design, we investigated the effects of phaseadvancing bedtime on sleep quality and duration among early-morning shift workers.

Methods The sleep-wake patterns of 16 healthy volunteers who work shifts with start times between 04.00 07.30 hours were recorded by wrist actigraphy and sleep diary for two weeks. In week 1 , we assessed the habitual sleep-wake pattern. In week 2, participants were required to advance their bedtime to 10 hours before the start of the morning shift. Subjective sleepiness was assessed three times each day.

Results Total sleep time was longer (1.4 hours) for sleep episodes that occurred prior to days off from work compared to those prior to early-morning shifts. The intervention resulted in bedtime being advanced by 1.5 hours and total sleep time being increased by 1.0 hour, without changes to the latency, efficiency or quality of sleep, or activity level during the sleep episode. A small reduction in daytime sleepiness was found during the intervention phase for assessments taken just prior to bedtime.

Conclusions Sleep duration among early-morning shift workers is substantially truncated due to their work schedules. When participants were instructed to advance their bedtime for one week, these individuals were able to increase sleep duration without affecting sleep quality. We suggest longer term studies testing the efficacy of sleep extension as a low-cost, behavioral intervention for improving health and safety outcomes among earlymorning shift workers.

Key terms actigraphy; circadian; shift work; wake maintenance zone.

Modern society is changing rapidly in terms of economic strategies, organizational, and individual behavior (1). While shift work may be broadly defined (2), work start times that fall between $04.00-08.00$ hours are typically classified as early-morning shift work (3-5). Recent data indicate that a considerable proportion of employees in Western countries are engaged in early-morning shift work: over a third of the Australian workforce starts work by 07.30 hours (6), 32.7\% of the Swedish work force regularly starts work between $03.00-05.30$ hours (7), and about $20 \%$ of the total workforce in the USA starts work between 02.30-07.00 hours (8).

Due to the temporal irregularity of shift work schedules, disturbance of the sleep-wake cycle is a common complaint (9-12). Sleep duration prior to an early-morning shift is often limited to 5-6 hours $(13,14)$. Reduction in the nocturnal sleep duration of early-morning shift workers results from premature termination of sleep due to the inherently early work start time without a concomittent advancement of bedtime (15). Among train drivers with start times between $04.30-09.00$ hours, a near linear relationship between sleep duration and start time has been reported with approximately 0.7 hours more sleep for every 1 hour the shift was delayed (16). The shortest sleep duration ( $~ 5$ hours) was observed for work shifts that began before 04.30 hours. Similarly, in a study of 500 shift workers, workers slept on average 5.9 hours between successive morning shifts and 8.4 hours between successive afternoon shifts (17). Sleepiness ratings were also consistently higher among those working morning compared to afternoon shifts. Among charter and air-freight pilots on short-haul routes, approximately 30 minutes of sleep was lost for every hour that duty was advanced prior to 09.00 hours (18). In addition to

\footnotetext{
${ }^{1}$ School of Psychology and Psychiatry, Monash University, Victoria, Australia

Correspondence to: Shantha MW Rajaratnam, School of Psychology and Psychiatry, Monash University, Building 17, Clayton Campus, Victoria 3800, Australia. [E-mail: shantha.rajaratnam@med.monash.edu.au]
} 
reduced sleep duration, the quality of sleep of earlymorning shift workers is impaired. Sleep onset latency is longer on the nights prior to morning shifts, with a corresponding reduction in sleep efficiency (19-21).

The observed reduction in nocturnal sleep duration among morning shift workers may be explained in terms of the endogenous circadian rhythm of sleep propensity $(12,15,20)$. The "forbidden zone" for sleep (22), or wake maintenance zone (23), is temporally positioned a few hours prior to the onset of the nocturnal sleep period. During this time, sleep propensity is markedly reduced and as a result sleep latency is increased and sleep efficiency is low (24). The reduced propensity for sleep at this time is thought to result from a strong wakepromoting signal generated by the endogenous circadian pacemaker (25). This suggests that conscious efforts to advance shift workers' bedtimes to earlier hours may not necessarily result in earlier sleep onset due to the wake maintenance zone.

Aside from circadian influences, social factors can also play a role in the sleep-wake behavior of morning shift workers. Despite the move to a 24-hour society, many aspects of our society are still primarily structured around a diurnal schedule $(26,27)$. Consequently, the tendency for bedtimes to remain unchanged among early-morning shift workers may be due in part to these individuals prioritizing engagement in social activities and fulfilling domestic roles (28-30).

Truncated night sleep, as observed among earlymorning shift workers, results in partial sleep deprivation. A "sleep debt" is incurred due to the accumulated disparity of the morning shift worker's sleep need and the prolonged (or chronic) sleep restriction (31). In the short term, chronic sleep restriction is likely to result in excessive daytime sleepiness (32-34) causing increased risk of sleep-related motor-vehicle crashes and workrelated injuries and errors $(17,35,36)$. In the long term, chronic sleep restriction is associated with increased risk of adverse health outcomes including obesity, diabetes, hypertension, and cardiovascular disease $(37,38)$. Moreover, shift work is associated with a number of adverse health consequences. Epidemiological evidence suggests that shift work is associated with gastrointestinal, immunological, and cardiovascular disease, as well as diabetes, metabolic disturbances, and increased risk of cancer (39-42). At least some of these adverse outcomes may be attributed to the chronic sleep restriction experienced by shift workers.

Evidence-based interventions to improve sleep quality and duration among early-morning shift workers are needed. The primary aim of this preliminary study was to investigate the effects of a short-term (1 week) intervention (consisting of advancing bedtime with no change in wake-up time) on sleep quality and duration among individuals who work shifts with early start times

(04.00-07.30 hours). We hypothesized that the overall total sleep time would be increased by the intervention, but that sleep latency would be increased and sleep efficiency decreased. To examine whether early-morning shift workers were adjusting their sleep-wake habits on days off from work, we compared sleep timing and duration on the nights preceding early-morning shifts and those preceding days off from work. Finally, we examined the effects of the intervention on self-reported daytime sleepiness.

\section{Methods}

\section{Participants}

A total of 23 participants expressed interest in participating in the study after initial eligibility was determined, of which 3 withdrew from the study prior to commencing baseline data collection. In total, 20 participants were enrolled in the study. Of these, 4 were discontinued or excluded from analyses due to equipment malfunction or lack of compliance with the protocol. To be deemed to be compliant, participants needed to advance their bedtime to 10 hours [acceptable range \pm 1 hour] prior to the start of the shift, on $90 \%$ of the nights prior to the early-morning shift during the intervention phase. The remaining 16 healthy volunteers ( 7 males, 9 females) were aged 20-52 years [mean 35.44 years, standard deviation (SD) 10.03]. Participants reported commencing their morning shift between 05.00-07.30 hours (mean 06.56 hours, SD 0.57 hours) with a mean shift duration of 8.57 hours (SD 0.58 hours). Of the 16 participants, 2 were divorced, 10 were in current marital or partnership relationships, and 4 reported not being in a relationship. The sample obtained included the following sectors: manufacturing $(\mathrm{N}=6)$, customer service/management $(\mathrm{N}=4)$, nursing $(\mathrm{N}=3)$, building/construction $(\mathrm{N}=2)$, and public service $(\mathrm{N}=1)$.

The morningness-eveningness questionnaire (43) revealed that eight participants were intermediate type and seven were moderately morning type (data from one participant were not available). Individuals were excluded if they reported a history of medical, psychiatric, or sleep disorder or regularly took medication known to cause sleepiness or fatigue.

Participants were recruited through posters and electronic notices distributed in organizations employing shift workers and displayed in public areas in the Melbourne metropolitan area. The study was conducted between August-December 2009. Participants were paid AU\$210 for completing the study.

Written informed consent was obtained. Monash University Human Research Ethics Committee approved the protocol. 


\section{Procedure}

The study used a within-subjects design and was conducted in the participants' normal environment over two consecutive weeks: the baseline phase in week 1 and the intervention phase in week 2. During the baseline phase, participants' habitual sleep-wake patterns, including daytime naps, were monitored for seven days. At the conclusion of the baseline phase, participants were contacted by telephone and were reminded to advance their bedtime for the intervention phase. During the intervention phase, on evenings prior to an early-morning shift, participants were required to advance their bedtime to occur 10 hours ( \pm 1 hour) before the start of morning shift. This was done to provide the opportunity for 8 hours time in bed. During the intervention phase, participants were asked to refrain from daytime napping so as to avoid any potential deleterious effects of daytime naps on nocturnal sleep. Furthermore, participants were encouraged to contact researchers should unforeseeable adverse events occur during the study.

\section{Materials}

A standard sleep diary was completed and an actigraph (Actiwatch L, Respironics Inc, Bend, OR, USA) was worn for two weeks on the non-dominant wrist. Subjective sleep quality was measured using a $\sim 100 \mathrm{~mm}$ horizontal line (visual analogue scale) anchored with "best sleep for a long time" and "worst sleep for a long time". Participants were required to evaluate the quality of their nocturnal sleep along the continuum by placing a mark along the line (44). Karolinska Sleepiness Scales (KSS) (45) were completed daily at three time points: 4 hours after wake time, 4 hours before bedtime, and immediately before bedtime. These time points were selected to minimize the impact of sleep inertia (46) in the morning assessment and to assess sleepiness in the subjective evening hours and just prior to bedtime. Participants completed the morningness-eveningness questionnaire to assess sleep timing preference (43).

\section{Data analysis}

In the primary analysis, actigraphy and sleep diary data only from the nights prior to early-morning shifts were included. For participants who completed an unequal number of shifts in week 1 and 2, data were matched to the minimum number of morning shifts undertaken in both weeks to maintain a consistent number for comparison. If, for example, 3 and 5 morning shifts were worked in weeks 1 and 2 respectively, only data from the first 3 morning shifts in week 2 were analyzed. Of the 16 participants, 7 worked a minimum of 3 mornings shifts, 2 worked 4 morning shifts and 7 worked 5 morning shifts during weeks 1 and 2 of the study. Actigraphy data was extracted and analyzed using Actiware 3.4 software (Mini Mitter, Respironics Inc, Bend, OR, USA), as in previous studies (47). The software automatically scores 1-minute epochs as sleep or wake-based on the number of activity counts within the epoch. The threshold for determining an epoch as wake was set at 40 activity counts (medium sensitivity). The software scored sleep onset as the first epoch in a series of $\geq 10$ minutes in which $\leq 1$ epoch contained measured activity. Sleep offset was determined as the last immobile epoch in a series of $\geq 10$ minutes, in which $\leq 1$ epoch contained measured activity. Compared to the gold-standard polysomnography, actigraphy is regarded to be moderately valid and reliable in defining sleep and wake states (48), although we note there is limited evidence to support the validation of specific sleep scoring algorithms (49).

Sleep parameters were determined using standard procedures (50). These included total sleep time as the primary outcome and sleep latency, sleep efficiency, and mean activity level as secondary outcomes. Lights-out time was determined from sleep diary and wake time was determined from sleep diary and actigraphy. Total sleep per sleep-wake cycle was calculated as the sum of total sleep time for the nocturnal sleep period and any naps occurring between the successive two morning shifts. For each participant, mean values were calculated separately for the baseline and intervention phases for each for the following sleep parameters: (i) bed and rise times, (ii) total sleep time, (iii) sleep onset latency, (iv) sleep efficiency, and (v) mean activity level. Mean activity level is an indication of the magnitude of activity during sleep and an indirect measure of sleep quality $(48,51)$. For daytime sleepiness, mean KSS scores were calculated for each of the three time points across the wake episodes during baseline and intervention phases. Sleep parameters for baseline and intervention phases were compared using repeated measures t-tests via SPSS statistics software, version 17.0 for Windows (SPSS Inc, Chicago, IL, USA).

The minimum sample size required to detect a significant effect in the primary outcome was set to 10 based on our previous laboratory study (51) demonstrating that a 5-hour phase advance of the sleep-wake cycle resulted in a $20 \%$ decrease in sleep efficiency $(\mathrm{N}=8, \mathrm{P}=0.007)$.

\section{Results}

\section{Habitual sleep patterns}

In the baseline phase, rise time occurred 3.0 hours earlier and total sleep time was 1.5 hours shorter during sleep episodes occurring prior to early-morning shifts compared to those occurring prior to days off $(\mathrm{P}<0.001)$ (see table 1 
and figures 1 and 2). In contrast, bedtime did not change. Likewise, sleep quality, sleep efficiency, sleep latency and mean activity levels did not differ between early-morning shifts and days off from work. Data from one participant were excluded from this analysis as no days off from work were reported during the baseline phase.

Effects of the intervention on sleep duration and quality

Bedtime occurred on average 1.5 hours earlier during the intervention compared to the baseline phase $(\mathrm{P}<0.001)$ (see table 2 and figures 1 and 2). Total sleep time was increased by 1.0 hour $(\mathrm{P}<0.001)$ during the intervention phase. Mean activity levels also increased during the intervention phase $(\mathrm{P}=0.02)$. Sleep efficiency and sleep latency did not differ between the baseline and intervention phases (see table 2).
Effects of the intervention on daytime sleepiness

Subjective sleepiness scores (assessed with the KSS) showed a modest decrease at each of the three time points (four hours after wake time, four hours before bedtime and immediately before bedtime) from baseline to the intervention phase. A significant decrease in sleepiness (1.1 units on KSS) was found only for the assessment taken immediately before bedtime $(\mathrm{P}<0.01)$ (see table 2 ).

\section{Discussion}

This study examined the effects of a short-term (1 week) intervention, consisting of advancement of bedtime, on the duration and quality of sleep of early-morning shift

Table 1. Sleep parameters assessed during the baseline phase in sleep episodes occurring prior to early-morning shifts and prior to days off from work. Mean, standard deviation (SD), t- and P-values, and $\eta^{2}$ (effect size) are shown. Sample size $=15$ for all parameters

\begin{tabular}{|c|c|c|c|c|c|c|c|}
\hline & \multicolumn{2}{|c|}{ Early shifts } & \multicolumn{2}{|c|}{ Days off } & \multirow[t]{2}{*}{$\mathrm{t}$} & \multirow[t]{2}{*}{ P-value } & \multirow[t]{2}{*}{$\eta^{2}$} \\
\hline & Mean & SD & Mean & SD & & & \\
\hline \multicolumn{8}{|l|}{ Sleep diary } \\
\hline Bedtime (clock time) & $22: 26$ & $00: 39$ & $22: 43$ & 03:04 & -0.39 & 0.7 & 0.01 \\
\hline Rise time (clock time) & 05:35 & $00: 48$ & $08: 37$ & 01:19 & -9.28 & $<0.001$ & 0.86 \\
\hline Sleep quality & 5.4 & 0.9 & 5.3 & 1.6 & 0.41 & 0.7 & 0.01 \\
\hline \multicolumn{8}{|l|}{ Actigraphy } \\
\hline Total sleep time (hours) & 5.7 & 0.9 & 7.2 & 1.3 & -5.3 & $<0.001$ & 0.67 \\
\hline Sleep efficiency (\%) & 80.2 & 5.7 & 78.5 & 9.2 & 1.0 & 0.3 & 0.07 \\
\hline Sleep latency (minutes) & 21.6 & 19.9 & 20.4 & 15.7 & 0.2 & 0.8 & 0.004 \\
\hline Mean activity level & 16.0 & 7.1 & 16.3 & 8.0 & -0.2 & 0.8 & 0.003 \\
\hline
\end{tabular}

Clock time (hours)

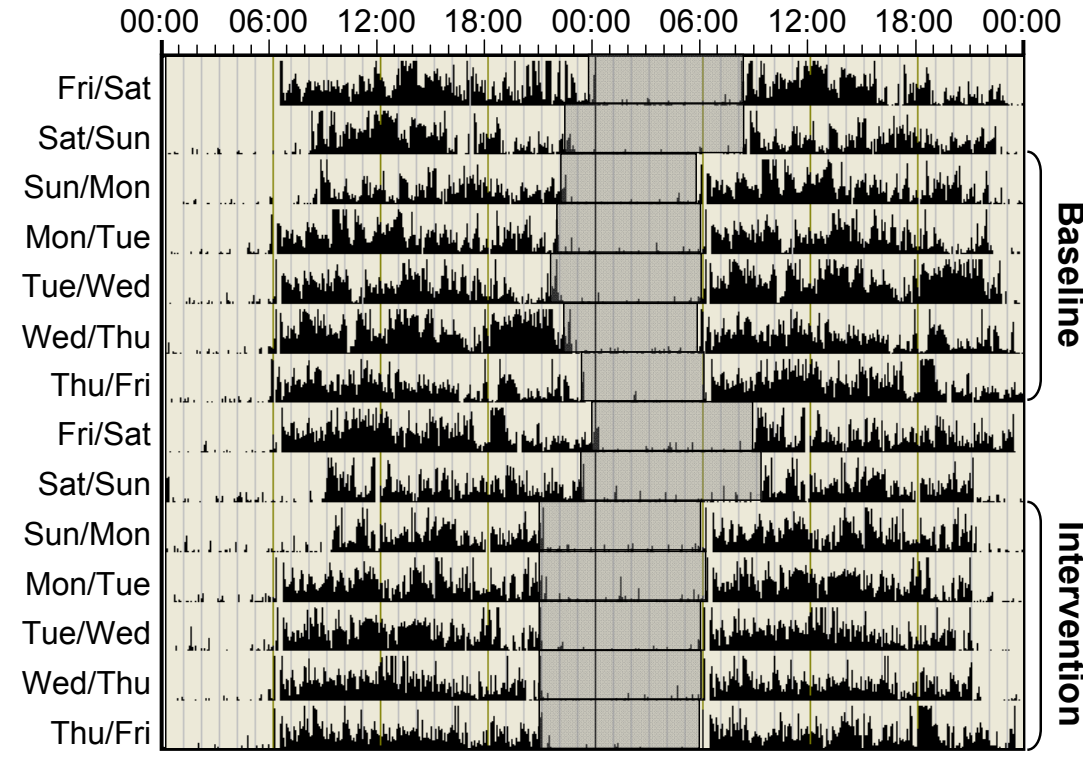

Figure 1. Actigraph record for one representative participant illustrating the phase advance in bedtime during the intervention phase, and the extended sleep episodes during days off from work. Successive days are shown beneath each other, and clock time (hours) is shown on the horizontal axis. Data are double plotted, meaning that 48 hours of data are shown on the horizontal axis. The baseline and intervention phases are marked, with two days off from work occurring between each phase. Sleep episodes (time in bed), assessed using sleep diaries, are depicted with gray-shaded bars. 
workers. We found that total sleep time was increased in response to the intervention. We also confirmed that sleep duration was substantially increased on the nights before days off from work compared to those before early-morning shifts.

In the baseline phase, sleep duration prior to morning shifts was just $<6$ hours, which is consistent with previous field studies $(15,14)$. One of the primary causes of the reduction in total sleep time is early termination of the sleep period due to early start time of the scheduled morning shift $(13,16)$. During the baseline phase, participants did not advance their bedtime to compensate for the early rise time associated with the morning shift. These individuals extended their sleep duration on days off from work by delaying their rise time, suggesting that they are attempting to "catch-up" on sleep when possible.
These findings indicate that early-morning shift workers are exposed to chronic sleep restriction during periods of successive early-morning shifts.

The earlier bedtimes during the intervention phase compared to baseline demonstrate that the intervention was successfully implemented. Bedtimes were advanced on average by 1.5 hours compared to baseline, in contrast to the lack of difference in rise times. As a consequence of earlier bedtimes, a gain of approximately 1 hour in total sleep time was also observed. Contrary to what was expected, increased level of sleep disturbance (indicated by increased sleep latency, decreased sleep efficiency, and increased activity level during the nocturnal sleep episode) did not occur during the intervention phase. This hypothesis was based on previous findings that sleep latency is increased and sleep efficiency

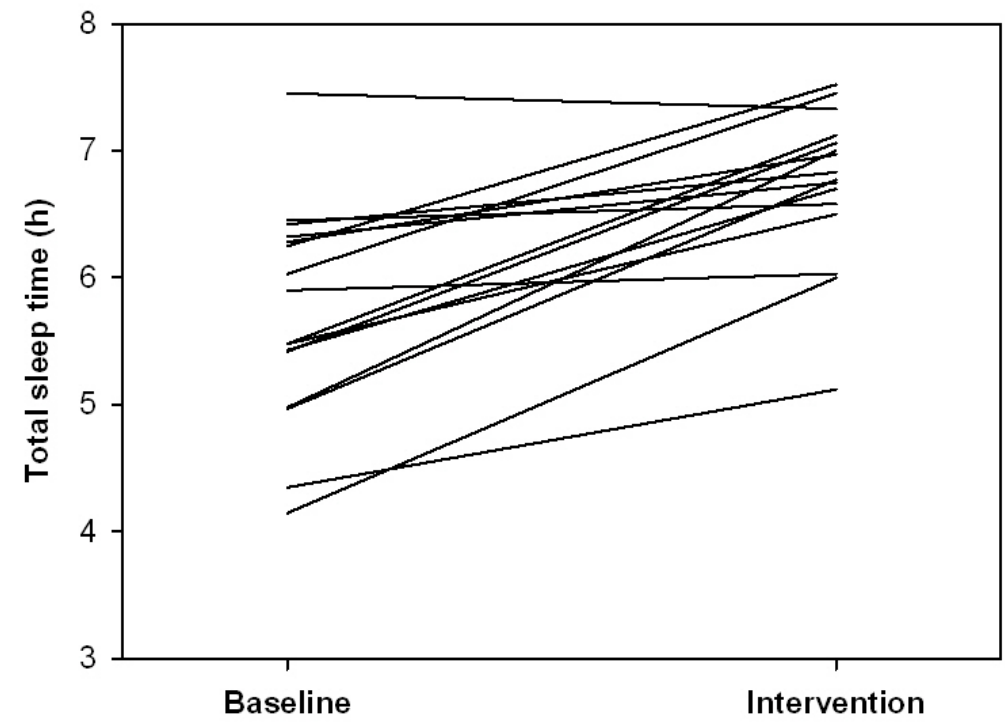

Figure 2. Total sleep time (hours) for individual participants during the baseline and intervention phases.

Table 2. Sleep parameters and subjective sleepiness during baseline and intervention phases. Mean, standard deviation (SD), t- and Pvalues and $\eta^{2}$ (effect size) are shown. Sample size=16 for all parameters. [KSS=Karolinska Sleepiness Scale.]

\begin{tabular}{|c|c|c|c|c|c|c|c|}
\hline & \multicolumn{2}{|c|}{ Baseline phase } & \multicolumn{2}{|c|}{ Intervention phase } & \multirow[t]{2}{*}{$\mathrm{t}$} & \multirow[t]{2}{*}{ P-value } & \multirow[t]{2}{*}{$\eta^{2}$} \\
\hline & Mean & $\mathrm{SD}$ & Mean & SD & & & \\
\hline \multicolumn{8}{|l|}{ Sleep diary } \\
\hline Bedtime (clock time) & $22: 29$ & $00: 39$ & $21: 00$ & $0: 35$ & 8.7 & $<0.001$ & 0.84 \\
\hline Rise time (clock time) & 05:36 & $00: 47$ & 05:36 & $00: 46$ & 0.1 & 0.9 & 0.001 \\
\hline Sleep quality & 5.6 & 1.3 & 6.0 & 1.3 & -1.3 & 0.2 & 0.10 \\
\hline \multicolumn{8}{|l|}{ Actigraphy } \\
\hline Total sleep time (hours) & 5.7 & 0.9 & 6.7 & 0.6 & -5.9 & $<0.001$ & 0.70 \\
\hline Sleep per 24 hours (hours) & 5.8 & 0.8 & 6.7 & 0.6 & -6.0 & $<0.001$ & 0.70 \\
\hline Sleep efficiency (\%) & 80.3 & 5.5 & 78.5 & 6.4 & 1.1 & 0.3 & 0.08 \\
\hline Sleep latency (minutes) & 21.5 & 19.2 & 31.8 & 15.8 & -1.7 & 0.1 & 0.16 \\
\hline Mean activity level & 15.6 & 7.0 & 18.9 & 10.2 & -2.7 & 0.02 & 0.33 \\
\hline \multicolumn{8}{|l|}{ Subjective Sleepiness (KSS) } \\
\hline Four hours after rise time & 3.48 & 1.09 & 3.19 & 0.95 & 1.7 & 0.1 & 0.16 \\
\hline Four hours before bedtime & 4.20 & 1.55 & 3.92 & 1.12 & 1.2 & 0.3 & 0.08 \\
\hline Immediately before bedtime & 6.54 & 1.59 & 5.48 & 1.32 & 3.1 & $0.01^{\mathrm{a}}$ & 0.38 \\
\hline
\end{tabular}


decreased when the sleep episode coincides with the circadian wake maintenance zone (24). Participants in the present study were able to fall asleep during the evening hours, the period in which we estimated the wake maintenance zone to occur. It is suggested that due to the accumulated homeostatic sleep pressure following chronic sleep restriction, participants were able to "over-ride" the circadian drive for wakefulness in the evening hours. We note, however, that the time course for recovery from chronic sleep restriction is relatively rapid (52), and therefore one may expect that this effect would dissipate after the first day of the intervention. Alternatively, it may be that the advance in bedtime did not cause the sleep episode to occur during the wake maintenance zone. Laboratory studies have demonstrated that the circadian drive for wakefulness peaks approximately 16 hours after the time the core body temperature rhythm reaches its minimum (24). Among individuals who are appropriately synchronized to the 24-hour day, the wake maintenance zone, or "forbidden zone" for sleep, therefore occurs at around 20.00-22.00 hours (22). Given that participants in our study were early-morning shift workers, it is likely that increased early-morning light exposure due to earlier wake time would phase advance the circadian pacemaker (53-56). In turn, this may result in the wake maintenance zone occurring at an earlier clock time. As we did not assess circadian phase in the present study, it was not possible to test this hypothesis.

As a secondary aim, the effect of advancing bedtime on subjective daytime sleepiness was investigated. This was deemed to be an important consideration in developing interventions for shift workers, in view of the significant neurobehavioral impairments and increased risk of accidents and injuries reported among such individuals (57). We found a modest decrease in sleepiness only in the evening during the intervention phase. The modest improvement in sleepiness at that time, and the lack of effect at other times, may be due to impairment in the individual's ability to self-assess his or her sleepiness level. A study assessing the relationship between habitual sleep duration and accuracy in self-reported alertness revealed that "short" sleepers may have reduced capacity to assess physiological sleepiness, as they tended to underestimate sleepiness (58). Other possible reasons for the lack of robust improvements in sleepiness include the following: (i) the relatively short duration of the intervention (1 week) and the limited timepoints for assessment; (ii) interindividual differences in bedtimes resulting in variability in the times of day (and potentially the circadian phase) at which the second and third sleepiness assessments were completed; (iii) inadequate sample size to yield sufficient statistical power to detect improvements in daytime sleepiness secondary to a 1-hour increase in total sleep time; and (iv) the uncontrolled conditions in which sleepiness was self-assessed, and hence the potential for situational factors to compensate for high sleepiness levels (59). Finally, a 1-hour earlier work start time was associated with reduced daytime alertness (60) and poorer psychological and physical health (61) among industrial shift workers, raising the possibility that delaying work start times may be a more effective way to improve sleepiness than advancing bedtime.

The limitations of the study should be noted. While the sample size was relatively small $(\mathrm{N}=16)$, we were able to detect an increase in total sleep time with medium effect size. Due to the limited duration of the intervention (1 week), and the lack of evaluation of its impact on social variables, it would be premature to suggest that advancing bedtime should be recommended to early-morning shift workers to improve their quality and duration of sleep in the long-term. Future studies would benefit from extending the protocol to collecting data on days off after the intervention phase. Total sleep time on days off following the intervention phase may indicate whether or not a sleep debt accrued during the intervention phase. A longer term study will also assess the practicality of maintaining an advanced sleep schedule. Furthermore, the current study did not include a control group. Future studies could benefit from the inclusion of a non-treated, randomized control group. We did not assess sleepiness immediately upon awakening, as we anticipated that sleep inertia would impair alertness at this time (46). We acknowledge that an alternative view is that it may be of particular interest to assess whether the intervention improved alertness at this time of increased sleepiness. Finally, the social impact of the intervention was not examined in the current study. In translating these findings, we acknowledge that advancing sleep time will result in other activities including social and family obligations being sacrificed in preference for sleep $(30,62)$. The long term impact of changes to time available for these activities should be examined.

Notwithstanding the above limitations, the present study provides evidence that, at least in the short term, early-morning shift workers are able to obtain more sleep if they advance their bedtime to an earlier hour. To our knowledge, no previous study has examined the effects of advancing bedtime on sleep quality and duration among early-morning shift workers. Based on the assessment of sleep during the baseline phase, it is evident that the sleep duration of early-morning shift workers is curtailed. As chronic sleep restriction is associated with a number of adverse health, safety, and performance outcomes (63), it is important that evidence-based interventions are developed for earlymorning shift workers. The present study provides the basis for a longer term study examining the efficacy 
of sleep extension (by phase advancing bedtime), as a low-cost, behavioral intervention for improving health and safety outcomes among early-morning shift workers. Early-morning shift workers may also benefit from educational programs about the importance of obtaining adequate sleep, and strategies to advance the timing of their sleep.

\section{Acknowledgements}

The authors express gratitude to Mrs Josefine Antoniades and Mrs Nicole Hennessy for their assistance with the study. The study was supported by the Australasian Sleep Trials Network (National Health and Medical Research Council Enabling Grant \#343020).

\section{References}

1. Rajaratnam SM, Arendt J. Health in a 24-h society. Lancet. 2001;358(9286):999-1005.

2. Grosswald B. The effects of shift work on family satisfaction. Families in Society-the Journal of Contemporary Human Services. 2004;85(3):413-423.

3. Allan C, Brosnan P, Walsh P. Non-standard working-time arrangements in Australia and New Zealand. Int J Manpower. 1998;19(4):234-249.

4. Harvey A S, Fisher K, Gershuny JI, Akbari SAH. Examining Working Time Arrangements Using Time Use Survey Data. Colchester (UK): Institute for Social and Economic Research, University of Essex; 2000.

5. Driscoll TR, Grunstein RR, Rogers NL. A systematic review of the neurobehavioural and physiological effects of shiftwork systems. Sleep Med Rev. 2007;11(3):179-94.

6. Venn D. Work timing arrangements in Australia in the 1990s: evidence from the Australian Time Use Survey [dissertation]. Melbourne (Australia): Department of Economics, the University of Melbourne; 2004.

7. Akerstedt T, Kecklund G. Early morning work - Prevalence and sleep/wake problems. Proceedings of the 19th International Symposium on Shiftwork and Working Time on 2-6 August, 2009; Venice, Italy; 2009. p.24

8. U.S Bureau of Labor Statistics. Washington DC. Workers in flexible and shift schedule in 2004 Summary [Internet]; [updated July 2005, cited 20 July 2009]. Available from: www. bls.gov/news.release/flex.nr0.htm.

9. Knauth P, Rutenfranz J. Duration of sleep related to the type of shift work. In: Reinberg A, Vieux N, Andlauer P, editors. Night and Shift Work: Biological and Social Aspects. Oxford: Pergamon Press; 1981. p161-168.

10. Härmä M. Sleepiness and shiftwork: individual differences. J Sleep Res. 1995;4 Suppl 2: 57-61.
11. Åkerstedt T. Shift work and disturbed sleep/wakefulness. Sleep Med Rev. 1998;2(2):117-128.

12. Akerstedt T. Shift work and disturbed sleep/wakefulness. Occup Med (Lond). 2003;53(2):89-94.

13. Pilcher JJ, Lambert BJ, Huffcutt AI. Differential effects of permanent and rotating shifts on self-report sleep length: a meta-analytic review. Sleep. 2000;23(2):155-63.

14. Kawada T, Shimizu T, Fujii A, Kuratomi Y, Suto S, Kanai T, et al. Activity and sleeping time monitored by an accelerometer in rotating shift workers. Work. 2008;30(2):157-60.

15. Kecklund G, Akerstedt T. Effects of timing of shifts on sleepiness and sleep duration. J Sleep Res. 1995;4(S2):47-50.

16. Ingre M, Kecklund G. Sleep length as a function of morning shift-start time in irregular shift schedules for train drivers: self-rated health and individual differences. Chronobiol Int. 2008;25(2):349-58.

17. Folkard S, Lombardi DA, Tucker PT. Shiftwork: safety, sleepiness and sleep. Ind. Health 2005;43(1):20-3.

18. Spencer M, Montgomery J. Sleep patterns of aircrew on charter / air haulage routes. PLSD Report No PSLD/CHS5/ CR/96/082. Hampshire (UK): Defence Evaluation \& Research Agency. 1997.

19. Åkerstedt T, Kecklund G, Knutsson A. Spectral analysis of sleep electroencephalography in rotating three-shift work. Scand J Work Environ Health 1991;17(5):330-6.

20. Åkerstedt T. Work hours, sleepiness and the underlying mechanisms. J Sleep Res. 1995; 4 Suppl 2:15-22.

21. Kecklund, G., T. Åkerstedt, Lowden A. Morning work: Effects of early rising on sleep and alertness. Sleep 1997;20(3):215223.

22. Lavie P. Ultrashort sleep-waking schedule. III. 'Gates' and 'forbidden zones' for sleep. Electroencephalogr Clin Neurophysiol. 1986;63(5):414-25.

23. Strogatz SH, Kronauer RE, Czeisler CA. Circadian regulation dominates homeostatic control of sleep length and prior wake length in humans. Sleep. 1986;9(2):353-364.

24. Dijk D J, Czeisler CA. Contribution of the circadian pacemaker and the sleep homeostat to sleep propensity, sleep structure, electroencephalographic slow waves, and sleep spindle activity in humans. J. Neurosci. 1995;15(5 Pt 1):3526-38.

25. Fuller PM, Gooley JJ, Saper CB. Neurobiology of the sleep-wake cycle: sleep architecture, circadian regulation, and regulatory feedback. J Biol Rhythms. 2006;21(6):482-93.

26. Harrington JM. Health effects of shiftwork and extended hours of work. Occup Environ Med. 2001;58:68-72.

27. Monk TH, Folkard S. Making shift work tolerable. London: Taylor \& Francis; 1992.

28. Folkard S, Barton J. Does the 'forbidden zone' for sleep onset influence morning shift sleep duration? Ergonomics. 1993;36(1-3)85-91.

29. Reid K, Roberts T, Dawson D. Improving shiftwork management. Int J Occup Health Saf. 1997;13:439-450.

30. Wilson JL. The impact of shift patterns on healthcare professionals. J Nurs Manag. 2002;10(4):211-9.

31. Van Dongen HP, Maislin G, Mullington JM, Dinges DF. 
The cumulative cost of additional wakefulness: dose-response effects on neurobehavioral functions and sleep physiology from chronic sleep restriction and total sleep deprivation. Sleep. 2003;26(2):117-26.

32. Bonnet MH. Sleep deprivation. In: Kryger MH, Roth T, Dement WC, editors. Principles and practice of sleep medicine. Philadelphia: W.B.Saunders Company; 2000. p.53-71.

33. Axelsson J, Akerstedt T, Kecklund G, Lowden A. Tolerance to shift work-how does it relate to sleep and wakefulness? Int Arch Occup Environ Health. 2004;77(2):121-9.

34. Akerstedt T. Sleepiness and circadian rhythm sleep disorders. Sleep Med Clin. 2006;1:17-30.

35. Marcus CL, Loughlin GM. Effect of sleep deprivation on driving safety in housestaff. Sleep. 1996;19(10):763-6.

36. Williamson AM, Feyer AM. Moderate sleep deprivation produces impairments in cognitive and motor performance equivalent to legally prescribed levels of alcohol intoxication. J Occup Environ Med. 2000;57(10):649-55.

37. Sigurdson K, Ayas N. The public health and safety consequences of sleep disorders. Canadian J Physiol Pharmacol. 2007.85:179-183.

38. Puttonen S, Harma M, Christer H. Shift work and cardiovascular disease: pathways from circadian stress to morbidity. Scand J Work Environ. Health. 2010;36(2):96-108.

39. Costa G. The impact of shift and night work on health. Appl. Ergonomics. 1996;27(1):9-16.

40. Costa G. Shift work and occupational medicine: An overview. Occup Med. 2003;53:83-88.

41. Knutsson A. Health disorders of shift workers. Occup Med (Lond). 2003;53(2):103-8.

42. Straif K, Baan R, Grosse Y, Secretan B, El Ghissassi F, Bouvard V, et al. Carcinogenicity of shift-work, painting, and fire-fighting. Lancet Oncol. 2007;8(12):1065-6.

43. Horne JA, Ostberg O. A self-assessment questionaire to determine morningness-eveningness in human circadian rhythms. Int J Chronobiology. 1976;4:97-110.

44. Curcio G, Casagrande M, Bertini M. Sleepiness: Evaluating and quantifying methods. Int J of Psychophysiol. 2001;21:251263.

45. Akerstedt T, Gillberg M. Subjective and objective sleepiness in the active individual. Int J Neurosci. 1990;52:29-37.

46. Jewett ME, Wyatt JK, Ritz-de Cecco A, Khalsa S, Dijk DJ, Czeisler CA. Time course of sleep inertia dissipation in human performance and alertness. J Sleep Res. 1999;8(1):1-8.

47. Knutson KL, Rathouz PJ, Yan LL, Liu K. Lauderdale DS. Intra-Individual Daily and Yearly Variability in Actigraphically Recorded Sleep Measures. Sleep. 2007;30(6):793-796.

48. Ancoli-Israel S, Cole R, Alessi C, Chambers M, Moorcroft $\mathrm{W}$, Pollak CP. The role of actigraphy in the study of sleep and circadian rhythms. Sleep. 2003;26(3):342-392.

49. Kripke DF, Hahn EK, Grizas AP, Wadiak KH, Loving RT,
Poceta JS. Wrist actigraphic scoring for sleep laboratory patients: algorithm development. J Sleep Res. Apr 7. 2010 [Epub ahead of print]

50. Morgenthaler T, Alessi C, Friedman L, Owens J, Kapur V, Boehlecke B, et al. Practice parameters for the use of actigraphy in the assessment of sleep and sleep disorders: an update for 2007. Sleep. 2007;30(4):519-29.

51. Rajaratnam SM, Polymeropoulos MH, Fisher DM, Roth T, Scott C, Birznieks G, et al. Melatonin agonist tasimelteon (VEC-162) for transient insomnia after sleep-time shift: two randomised controlled multicentre trials. Lancet. 2009;373(9662):482-491.

52. Åkerstedt T, Kecklund G, Ingre M, Lekander M, Axelsson J. Sleep Homeostasis During Repeated Sleep Restriction and Recovery: Support from EEG Dynamics. Sleep. 2009;32(2):217-22.

53. Honma K, Honma S. A human phase response curve for bright light pulses. Jpn J Psychiat Neurol. 1988;42:167-168.

54. Czeisler CA, Kronauer RE, Allan JS, Duffy JF, Jewett $\mathrm{ME}$, Brown EN, et al. Bright light induction of strong (Type O) resetting of the human circadian pacemaker. Science. 1989;244:1328-1333.

55. Minors DS, Waterhouse JM, Wirz-Justice A. A human phaseresponse curve to light. Neurosci Lett. 1991;133:36-40.

56. Khalsa SBS, Jewett ME, Cajochen C, Czeisler CA. A phase response curve to single bright light pulses in human subjects. J Physiol. 2003;549(Pt 3):945-52.

57. Barger LK, Lockley SW, Rajaratnum SM, Landrigan CP. Neurobehavioral, health, and safety consequences associated with shift work in safety-sensitive professions. Curr Neurol Neurosci Rep. 2009;9(2):155-64.

58. Richardson GS, Drake CL, Roehrs TA, Roth T. Habitual sleep time predicts accuracy of self-reported alertness. Sleep. 2002;25:A145-A145.

59. Anund A, Kecklund G, Kircher A, Tapani A, Akerstedt T. The effects of driving situation on sleepiness indicators after sleep loss: a driving simulator study. Ind Health. 2009;47(4):393-401.

60. Tucker P, Smith L, Macdonald I, Folkard S. Shift length as a determinant of retrospective on-shift alertness. Scand J Work Environ Health. 1998;24 Suppl 3:49-54.

61. Tucker P, Smith L, Macdonald I, Folkard S. The impact of early and late shift changeovers on sleep, health, and wellbeing in 8- and 12-hour shift systems. J Occup Health Psychol. 1998;3(3):265-75.

62. Shen J, Dicker B. The impacts of shiftwork on employees. Int J Hum Resour Man. 2008;19:392-405.

63. Banks S, Dinges DF. Behavioral and physiological consequences of sleep restriction. J Clin Sleep Med. 2007;3(5):519-28.

Received for publication: 7 May 2010 